\title{
Logros, dificultades y perspectivas de la Escuela de Formación Pedagógica
}

\author{
Carlos Bolívar Bonilla Baquero \\ Programa de Psicología \\ Coordinador EFP
}

Las principales universidades del mundo destinan importantes recursos humanos y financieros a la formación pedagógica de sus profesores. No los dedican sólo a la preparación disciplinar específica en un campo del conocimiento científico tecnológico. Pero no siempre fue así. La preocupación por el dominio profesoral del conocimiento científico era y es considerada hoy, en muchas partes, como prioritaria. Pero, después de la mitad del siglo $\mathrm{XX}$, empezó a romperse aquella errónea idea, según la cual, para ser profesor universitario basta con el dominio de un conocimiento disciplinar especializado. Es decir, se puso en cuestionamiento el supuesto: quien sabe algo, sabe enseñarlo, nacido, tal vez, en la profesionalizante universidad francesa del siglo XIX.

La enseñanza universitaria comenzó a convertirse en objeto de estudio $y$, en consecuencia, aparecieron múltiples alternativas de formación profesoral, que van desde cursos de pocas horas (esporádicos o permanentes), hasta especializaciones y maestrías. La Vicerrectoría Académica de la Universidad Surcolombiana (USCO) optó por una Escuela de Formación Pedagógica (EFP), a partir del actual Plan de Desarrollo Institucional, creada en el segundo semestre de 2015. En este escrito describo los logros, las dificultades y las perspectivas más relevantes de la EFP y sustento su trascendencia para el ejercicio profesoral, en coherencia con nuestro Proyecto Educativo Universitario (PEU). En Colombia, las universidades más reconocidas también tienen centros, departamentos, institutos, oficinas y otras dependencias encargadas de la formación pedagógica de sus profesores. A la EFP hemos invitado colegas de algunas de esas universidades (más adelante presento una relación detallada de los invitados y sus temas), responsables de dirigir procesos formativos, a compartir sus experiencias al respecto. En este contexto de preocupación por la formación pedagógica de los profesores universitarios, resalto la producción de un documento, en 2017, liderado por la Asociación Colombiana de Universidades (ASCUN), acerca del profesor universitario. En este texto es claro que ya no basta contar con un determinado conocimiento científico para definir al profesor, se defiende que en las actuales sociedades el profesor universitario es un profesional de altísima formación humana y pedagógica. Destaco en esta referencia el carácter de profesional del que enseña en la universidad y su correspondiente preparación humanística y pedagógica.

En concordancia con lo citado, la EFP se propuso ser un espacio institucional orientado a conseguir dos objetivos interdependientes: promover la asimilación profesoral del nuevo PEU y favorecer la reflexión pedagógica que haga posible su aplicación. Espacio inexistente antes de la creación de la EFP. Para lograr estos objetivos adoptamos la estrategia de combinar los aportes de expertos de reconocida trayectoria académica en diversos campos de la universitología, la pedagogía, las didácticas especiales y las ciencias sociales críticas, con las experiencias y conocimientos particulares de los profesores y las profesoras de nuestra universidad, participantes en la EFP.

La estrategia tuvo como principio pedagógico fundamental, vivir en la EFP un ambiente académico como el que el PEU y las pedagogías críticas plantean: flexible, pluralista, respetuoso, crítico, ameno y dialógico. No podíamos contradecirnos en esto, hablar de aperturas y 
novedades pedagógicas para la enseñanza universitaria, en un ambiente de rigidez curricular, frío, irreflexivo, dogmático y de monólogos soporíferos. Este es un logro destacado, experimentar en vivo que sí es posible sesionar alrededor de asuntos académicos, filosóficos, científicos, culturales, éticos y políticos, sin imposiciones ni exclusiones autoritarias. Que sí se pueden debatir y sustentar puntos de vista diferentes sobre tópicos de la vida universitaria y pactar acuerdos provisionales, sin irrespetos ni ofensas, con cordialidad. Experiencia esta de importantes impactos positivos en las aulas donde laboran las profesoras y los profesores de la EFP. Otro logro relevante es el de compartir lecturas pertinentes, escuchar y reflexionar sobre nuestras experiencias profesorales, a partir del diálogo franco con los invitados. Muchas veces los profesores inscritos en la EFP han reconocido que nunca se habían detenido a considerar temas relacionados con la pedagogía, la didáctica, las rúbricas y el currículo, o con conceptos como ética cívica, complejidad, epistemologías del Sur y Buen Vivir. Por lo general, son profesionales de la ingeniería, la economía, la enfermería, la contaduría, la ciencia política, la comunicación social y el derecho, entre otras. Profesiones y disciplinas en las cuales no se dedica tiempo al saber pedagógico, encargado del estudio de la enseñanza como vía de formación humana y todo lo que implica su aplicación escolarizada.

Para ilustrar mejor lo expuesto, en el siguiente cuadro presento la relación de invitados a la EFP, las fechas de su visita, sus temas y procedencias:

\begin{tabular}{|c|c|c|}
\hline FECHAS & TEMAS & INVITADO (A) \\
\hline $\begin{array}{l}28 \text { Agosto } \\
\text { de } 2015\end{array}$ & $\begin{array}{l}\text { Seminario-Taller: Prospectiva de la } \\
\text { Educación en Colombia. }\end{array}$ & $\begin{array}{l}\text { Dr. Alejandro Álvarez } \\
\text { Universidad Pedagógica } \\
\text { Nacional }\end{array}$ \\
\hline $\begin{array}{l}4 \text { de } \\
\text { Septiembre de } \\
2015\end{array}$ & $\begin{array}{l}\text { Seminario-Taller: ¿Quiénes somos } \\
\text { los maestros universitarios hoy? }\end{array}$ & $\begin{array}{c}\text { Dr. Alberto Martínez Boom } \\
\text { Universidad Pedagógica } \\
\text { Nacional }\end{array}$ \\
\hline $\begin{array}{l}17 \text { de } \\
\text { Septiembre de } \\
2015\end{array}$ & $\begin{array}{l}\text { Seminario-Taller: Evaluación en la } \\
\text { educación Superior. }\end{array}$ & $\begin{array}{l}\text { Dr. Fabio Jurado Valencia } \\
\text { Universidad Nacional de } \\
\text { Colombia }\end{array}$ \\
\hline $\begin{array}{l}2 \text { de } \\
\text { Octubre de } \\
2015\end{array}$ & $\begin{array}{c}\text { Conferencia: El oficio del maestro } \\
\text { en la Educación Superior }\end{array}$ & $\begin{array}{l}\text { Dr. Fernando Vásquez } \\
\quad \text { Rodríguez } \\
\text { Universidad de la Salle }\end{array}$ \\
\hline $\begin{array}{l}16 \text { de } \\
\text { Octubre de } \\
2015\end{array}$ & $\begin{array}{l}\text { Seminario-Taller: El sentido de la } \\
\text { Evaluación en la Educación } \\
\text { Superior. }\end{array}$ & $\begin{array}{l}\text { Dr. Daniel Bogoya } \\
\text { Universidad Nacional } \\
\text { de Colombia }\end{array}$ \\
\hline $\begin{array}{l}29 \text { de } \\
\text { Octubre de } \\
2015\end{array}$ & $\begin{array}{l}\text { Seminario-Taller: Hacia un nuevo } \\
\text { proyecto educativo y pedagógico. }\end{array}$ & $\begin{array}{c}\text { Dr. Marco Raúl Mejía } \\
\text { Investigador en Educación y } \\
\text { Pedagogía }\end{array}$ \\
\hline
\end{tabular}




\begin{tabular}{|c|c|c|}
\hline $\begin{array}{l}29 \text { de enero } \\
\text { de } 2016\end{array}$ & Conferencia: Equidad de género. & $\begin{array}{l}\text { Dra. Florence Thomas } \\
\text { Universidad Nacional de } \\
\text { Colombia }\end{array}$ \\
\hline $\begin{array}{l}19 \text { de febrero } \\
\text { de } 2016\end{array}$ & $\begin{array}{c}\text { Seminario-Taller: Contexto-USCO } \\
\text { Latinoamérica sobre el estado de la } \\
\text { Educación. }\end{array}$ & $\begin{array}{l}\text { Dr. Daniel Libreros } \\
\text { Universidad Nacional de } \\
\text { Colombia }\end{array}$ \\
\hline $\begin{array}{l}11 \text { de marzo } \\
\text { de } 2016\end{array}$ & $\begin{array}{c}\text { Seminario-Taller: Pensamiento } \\
\text { Complejo }\end{array}$ & $\begin{array}{l}\text { Dr. Carlos Eduardo } \\
\text { Maldonado } \\
\text { Universidad del Rosario }\end{array}$ \\
\hline $\begin{array}{l}15 \text { de abril } \\
\text { de } 2016\end{array}$ & $\begin{array}{c}\text { Seminario-taller: Ética cívica y } \\
\text { democracia deliberativa }\end{array}$ & $\begin{array}{c}\text { Dr. Óscar Mejía Quintana } \\
\text { Universidad Nacional de } \\
\text { Colombia }\end{array}$ \\
\hline $\begin{array}{l}3 \text { de junio } \\
\text { de } 2016\end{array}$ & $\begin{array}{l}\text { Seminario-Taller: la Rúbrica como } \\
\text { elemento formativo en el proceso de } \\
\text { evaluación. }\end{array}$ & $\begin{array}{c}\text { Dr. Fabio Jurado Valencia } \\
\text { Universidad Nacional de } \\
\text { Colombia }\end{array}$ \\
\hline $\begin{array}{l}29 \text { de julio } \\
\text { de } 2016\end{array}$ & Seminario-Taller: Multiculturalismo. & $\begin{array}{c}\text { Dra. Claudia del Pilar Vélez } \\
\text { Universidad Pedagógica } \\
\text { Nacional }\end{array}$ \\
\hline $\begin{array}{c}2 \text { de } \\
\text { septiembre } \\
\text { de } 2016\end{array}$ & $\begin{array}{l}\text { Seminario-Taller: Socio } \\
\text { construccionismo. }\end{array}$ & $\begin{array}{l}\text { Dra. Ángela María Estrada } \\
\text { Universidad de los Andes }\end{array}$ \\
\hline $\begin{array}{l}28 \text { de octubre } \\
\text { de } 2016\end{array}$ & Seminario Taller: Buen Vivir & $\begin{array}{c}\text { Dr. Adalid Contreras } \\
\text { Universidad Andina Simón } \\
\text { Bolívar }\end{array}$ \\
\hline $\begin{array}{l}02 \text { de diciembre } \\
\text { de } 2016\end{array}$ & $\begin{array}{l}\text { Seminario-Taller: El Maestro } \\
\text { empático- } \\
\text { La vida emocional en el aula. }\end{array}$ & $\begin{array}{l}\text { Dr. Guillermo Carvajal } \\
\text { Fundación Instituto } \\
\text { Colombiano de Psicoterapia } \\
\text { Integral }\end{array}$ \\
\hline
\end{tabular}




\begin{tabular}{|c|c|c|}
\hline $\begin{array}{l}17 \text { de febrero } \\
\text { de } 2017\end{array}$ & $\begin{array}{c}\text { Seminario-Taller: Teorías del } \\
\text { aprendizaje }\end{array}$ & $\begin{array}{l}\text { Dr. Jaime Parra Rodríguez } \\
\text { Universidad Javeriana }\end{array}$ \\
\hline $\begin{array}{l}24 \text { de marzo } \\
\text { de } 2017\end{array}$ & $\begin{array}{l}\text { Seminario-Taller: Diseño de } \\
\text { materiales educativos para la } \\
\text { docencia. }\end{array}$ & $\begin{array}{l}\text { Dra. Edna Manotas } \\
\text { Universidad del Norte }\end{array}$ \\
\hline $\begin{array}{l}28 \text { de abril } \\
\text { de } 2017\end{array}$ & $\begin{array}{l}\text { Seminario-Taller: El currículo en la } \\
\text { Educación Superior. }\end{array}$ & $\begin{array}{l}\text { Dra. María Constanza } \\
\text { Jiménez } \\
\text { Universidad Distrital }\end{array}$ \\
\hline $\begin{array}{l}19 \text { de mayo } \\
\text { de } 2017\end{array}$ & $\begin{array}{c}\text { Seminario-Taller: Evaluación del } \\
\text { Aprendizaje en la Educación } \\
\text { Superior. }\end{array}$ & $\begin{array}{l}\text { Dra. Martha Lorena Salinas } \\
\text { Universidad de Antioquia }\end{array}$ \\
\hline $\begin{array}{l}25 \text { de agosto } \\
\text { de } 2017\end{array}$ & $\begin{array}{l}\text { Seminario-Taller: Prácticas } \\
\text { significativas y proyectos de Aula. }\end{array}$ & $\begin{array}{l}\text { Dr. Mario Helmer Flórez } \\
\text { Director del Centro de } \\
\text { Investigaciones e Innovación } \\
\text { en los Negocios CIIN }\end{array}$ \\
\hline $\begin{array}{l}10 \text { de } \\
\text { noviembre } \\
\text { de } 2017\end{array}$ & $\begin{array}{c}\text { Seminario-Taller: La formación } \\
\text { pedagógica del maestro universitario. }\end{array}$ & $\begin{array}{l}\text { Dr. Andrés Klaus Runge } \\
\text { Universidad de Antioquia }\end{array}$ \\
\hline $\begin{array}{l}17 \text { de } \\
\text { noviembre } \\
\text { de } 2017\end{array}$ & $\begin{array}{l}\text { Seminario-Taller: Educación y } \\
\text { pensamiento complejo. }\end{array}$ & $\begin{array}{l}\text { Dr. Carlos Eduardo } \\
\text { Maldonado } \\
\text { Universidad del Rosario }\end{array}$ \\
\hline $\begin{array}{c}01 \text { de } \\
\text { diciembre } \\
\text { de } 2017\end{array}$ & $\begin{array}{l}\text { Seminario-taller Formación } \\
\text { del profesor } \\
\text { Universitario (y de los estudiantes) } \\
\text { y el método analítico en el aula. }\end{array}$ & $\begin{array}{l}\text { Dr. Juan Diego Lopera } \\
\text { Universidad de Antioquia }\end{array}$ \\
\hline $\begin{array}{l}23 \text { de febrero } \\
\text { de } 2018\end{array}$ & $\begin{array}{l}\text { Seminario-Taller: Sensibilización } \\
\text { artística para la enseñanza y } \\
\text { formación Universitaria }\end{array}$ & $\begin{array}{l}\text { Mg. Gustavo Bríñez } \\
\text { Universidad Surcolombiana }\end{array}$ \\
\hline
\end{tabular}




\begin{tabular}{|c|c|c|}
\hline $\begin{array}{l}16 \text { de marzo } \\
\text { de } 2018\end{array}$ & $\begin{array}{l}\text { Seminario-Taller: Pistas para pensar } \\
\text { la práctica docente universitaria }\end{array}$ & $\begin{array}{l}\text { Alfredo Manuel Ghiso } \\
\text { Universidad de Antioquia }\end{array}$ \\
\hline $\begin{array}{l}04 \text { de marzo } \\
\text { de } 2018\end{array}$ & $\begin{array}{c}\text { Seminario-Taller: Universidad } \\
\text { y pos - acuerdo }\end{array}$ & $\begin{array}{l}\text { Helberth Augusto Choachi } \\
\text { Universidad Pedagógica } \\
\text { Nacional }\end{array}$ \\
\hline $\begin{array}{l}7 \text { de septiembre } \\
\text { de } 2018\end{array}$ & $\begin{array}{c}\text { Seminario-Taller: Formación } \\
\text { Pedagógica }\end{array}$ & $\begin{array}{l}\text { Dr. Javier Faydad } \\
\text { Universidad del Valle }\end{array}$ \\
\hline $\begin{array}{l}05 \text { de octubre } \\
\text { de } 2018\end{array}$ & $\begin{array}{c}\text { Seminario-Taller: Pensamiento } \\
\text { crítico y dilemas éticos en la vida } \\
\text { Universitaria }\end{array}$ & $\begin{array}{c}\text { Dra. Isolda Arango-Álvarez } \\
\text { Lacanian Compass } \\
\text { Miami-USA }\end{array}$ \\
\hline $\begin{array}{c}02 \text { de } \\
\text { noviembre } \\
\text { de } 2018\end{array}$ & $\begin{array}{l}\text { Seminario-Taller: La pedagogía y la } \\
\text { enseñanza en la Educación Superior }\end{array}$ & $\begin{array}{l}\text { Dr. Jaime Saldarriaga } \\
\text { Universidad de Antioquia }\end{array}$ \\
\hline
\end{tabular}

Otro logro de especial carácter formativo es el de haber empezado, desde el segundo semestre de 2017, a exponer entre nosotros mismos nuestras experiencias didácticas y conocimientos disciplinares. Presentar ante nuestros colegas maneras de trabajar en el aula, temas de actualidad, dudas y aciertos, es enriquecedor. Algunos profesores y profesoras, como Martha Isabel Barrero, han ofrecido reflexiones acerca de sus recorridos por la enseñanza, cómo la empezaron y cómo la han transformado a lo largo de sus trayectorias. Otros han brindado disertaciones sobre temas de sus capacidades y vocaciones intelectuales, como Humberto Cabrera Zamora, quien disertó sobre los Quipus y la Yupana, saberes matemáticos ancestrales de los incas, mediante los cuales ejercían la contabilidad de sus propiedades y productos. Este profesor no sólo explicó el sistema numérico de la sociedad incaica, sino que lo relacionó con la escultórica agustiniana y la matemática actual, en una muestra clara de cómo practicar aquello del PEU, alusivo al diálogo entre epistemologías occidentales y del Sur.
El profesor Nelson López reconstruyó la vida y obra del célebre sociólogo colombiano Fals Borda, destacó sus principales aportes mediante la Investigación Acción Participativa y mostró su ejercicio profesoral como un referente digno de tener en cuenta, cuando nos preguntamos por cómo formar-nos en el oficio y el arte de la enseñanza superior. El colega Alberto Ducuara expuso las características, bondades y defectos del fordismo, en relación con los procesos educativos, lo cual animó un interesante debate sobre el tema. El coordinador de la EFP ofreció, en varias oportunidades, conferencias acerca del origen, la evolución, las características y los principales postulados de las pedagogías críticas y su conexión con el PEU. Y se preparan para el inmediato futuro otras participaciones. ¿En qué otro lugar de la universidad podemos compartir experiencias como estas?

Un logro adicional, de relevancia, lo constituye la publicación de dos libros, uno por año: 2016-2017 (trabajamos en la actualidad por la publicación del de 2018), con escritos muy variados pero que giran todos alrededor de la vida universitaria y la 
enseñanza. Con estos productos no sólo se visibiliza ante nuestra comunidad educativa lo que pensamos y hacemos en la EFP, sino que se construye una memoria colectiva de lo que trabajamos. Además, así se sistematizan y materializan las reflexiones de los profesores y las profesoras sobre diferentes aspectos de la formación en el contexto del PEU, lo cual configura una manera de producir saber pedagógico. Y no puedo dejar de resaltar que, para muchos colegas jóvenes, este ejercicio se convirtió en la apertura o iniciación de su labor de escritura. Condición inherente a la vida de todo aquel que se dedique a la enseñanza superior.

A propósito del libro proyectado para 2018, es necesario advertir que los grupos de trabajo de la Escuela desarrollan procesos investigativos en torno a sus respectivos ejes temáticos: las metodologías de enseñanza, la evaluación del rendimiento académico, la docencia universitaria y la investigación. Todos estos grupos están conformados por profesoras y profesores de diferentes programas y tienen el compromiso de entregar un artículo producto de sus indagaciones, al interior de la USCO, para alimentar el libro.

Otro notorio logro de la EFP es el de su pronunciamiento crítico ante procesos universitarios que afectan la vida académica de la institución. Tales son los casos de la reforma al Acuerdo 020 de 2005 y la medida administrativa inconsulta de visitar las aulas de clases para medir el desarrollo de los microdiseños curriculares y determinar, según esta lógica, si los profesores cumplimos o no lo programado. En el primer caso, aportamos una reflexión escrita concerniente a la concepción del profesor universitario en una universidad pública. Lo mismo que un conjunto de principios orientadores para la distribución de las horas en las agendas laborales. En el segundo caso, rechazamos su lógica empresarial del control cuantitativo, punitivo y milimétrico, sobre una actividad de predominio cultural comunicativo, no sujeta a algoritmos tecnocráticos.

El logro más reciente de la EFP es la determinación tomada para iniciar un ciclo de conversatorios entre los profesores y las profesoras de la Escuela, con estudiantes de distintas carreras, acerca de las concepciones sobre la universidad y el ejercicio de la enseñanza, los atributos distintivos de un buen profesor o profesora, las maneras de desarrollar los cursos, la evaluación tanto docente como estudiantil, los motivos de los conflictos y las claves de las buenas prácticas pedagógicas. Al momento de escribir este texto cumplimos ya el primero de estos conversatorios, con un positivo balance. Escuchamos a un grupo de 10 estudiantes y ellos nos escucharon a nosotros. Nos interrogaron por nuestras concepciones del profesor universitario y criticaron casos particulares de tratos autoritarios, carentes de eticidad, poco rigor académico, mínima motivación para el estudiante y mucha rigidez metodológica. Nosotros les hicimos ver que la enseñanza es un ejercicio complejo de responsabilidades compartidas, entre profesores y estudiantes, y que no siempre estos últimos obran de la mejor manera al momento de cumplir con sus deberes y de evaluar a sus docentes.

Lo más importante es que coincidimos en asuntos como la necesidad de aplicar los lineamientos pedagógicos del PEU, modificar el actual sistema de evaluación profesoral y seguir en la promoción constante de un diálogo público, respetuoso y sincero, para esclarecer los motivos de los conflictos y pactar los respectivos acuerdos. La experiencia fue muy bien valorada por unos y otros, se acordó perfeccionarla con la introducción de ejes temáticos más precisos para los nuevos conversatorios, una selección o convocatoria estudiantil focalizada por facultades y, luego de un tiempo, sistematizarla y publicarla. Esta es otra experiencia singular que nunca antes de la existencia de la EFP, habíamos podido desarrollar. Finalmente, en cuanto a los principales logros se refiere, la EFP ha conseguido su reconocimiento como un espacio pedagógico excepcional y potente para formar-nos, por parte de los profesores y las profesoras que desde su creación han acompañado sus sesiones. Se trata de un colectivo de unos 20 maestros y maestras, donde se integran colegas muy jóvenes y colegas veteranos, unidos a los 15 o 20 nuevos que cada semestre se inscriben. Todos ellos admiten que se trata de un lugar valioso para el fortalecimiento de sus procesos educativos. $\mathrm{Y}$ tengo que mencionar como logro fundamental, el apoyo institucional permanente de la Vicerrectoría Académica, dirigida por la profesora Isabel Cristina de Dussán. Por su tesón, 
no nos faltaron los recursos económicos básicos ni las directrices pertinentes desde el Consejo Académico. Todos estos logros han impactado de forma significativa y positiva el desempeño profesoral de quienes asisten a la EFP. Por supuesto que también tenemos dificultades. La primera de ellas es de carácter infraestructural. Aunque parezca extraño, la EFP no cuenta con un sitio fijo para sus sesiones. En muchas ocasiones, 24 horas antes de un encuentro o de la visita de un experto, no sabemos dónde reunirnos. Una alternativa para superar este obstáculo es insistir ante los encargados de la Oficina de Planeación, para que se considere a la Escuela como un curso regular que necesita de un aula permanente, durante el semestre.

La segunda barrera para nuestro desarrollo proviene de los jefes de Programa que no cooperan con sus profesores interesados en participar en la EFP. Estos jefes no cumplen con la política de no asignar cursos o compromisos laborales a los maestros, el viernes, día en que funciona la Escuela. Hemos perdido la participación de destacados colegas, por este motivo. La solución es cumplir con lo acordado en el Consejo Académico, para evitar la contradicción administrativa de pactar una política en un organismo y desconocerla en otro.

La tercera talanquera proviene de la dirección de la Editorial de la universidad, para la edición de nuestros libros. Desde una concepción cuadriculada de los requisitos para tramitar el ISBN, se ha pretendido aplicar las exigencias de los libros producto de investigación que llevan el sello de dicha Editorial, a los de la Escuela, que no lo son, ni reclaman su marca. Ignoro si para el libro tercero de 2018 se vuelva a intentar tal arbitrariedad, pues se torpedea así el esfuerzo de promover la escritura en los jóvenes profesores, no únicamente en la modalidad investigativa, sino también en géneros valiosos para los procesos de formar-nos, como la autobiografía, el ensayo, la crónica y la ponencia didáctica metodológica.

La cuarta piedra en el zapato es una paradoja y emerge de los propios inscritos en la EFP. Cada semestre tenemos algunos participantes que se inscriben de modo voluntario, reciben la asignación de las 4 horas semanales y no asisten de manera regular. Como muchos de nuestros estudiantes de pregrado, llegan tarde o no llegan a ciertas sesiones, se van temprano, no se integran a los grupos de trabajo, no leen los materiales enviados por los expertos y no participan en las deliberaciones colectivas. La impresión que dejan es la de tomar la Escuela como excusa para justificar las 4 horas en sus agendas, nada más. Cuando los interpelo por sus incumplimientos, siempre tienen pretextos muy "serios". Al terminar el semestre reportamos tales casos a las respectivas decanaturas sin que, al parecer, haya correctivos claros. En mi opinión, son comportamientos de incoherencia ética, que afectan el funcionamiento de la Escuela y la credibilidad de estos profesores.

No sería justo si, frente a la anterior dificultad, no destacara al grupo de profesores y profesoras que ha mantenido la constancia y su compromiso con el trabajo, durante los tres años de la EFP. Menos lo sería si olvidara que entre esos maestros y maestras hay un pequeño pero notable número de asistentes, unos de planta y otros catedráticos, que van a la Escuela sin el reconocimiento de horas en sus agendas. Esta es una clara demostración de convicción y satisfacción por el trabajo que allí hacemos.

La quinta valla a sortear tiene que ver con la composición de los miembros de la EFP, al empezar cada semestre. Consiste en tener, a un mismo tiempo, a los nuevos inscritos que por vez primera llegan, con los profesores y profesoras que ya llevan tres años. Aunque intento una inducción con el grupo nuevo (para lo cual diseñé una cartilla guía), no parece suficiente para ponerlos al día en todo lo tratado antes y, de extenderme, resultaría repetitivo para los antiguos. No hemos explorado una alternativa como la de crear dos grupos paralelos, por niveles o experiencia en la EFP, pero no es descartable.

Las perspectivas que vislumbro para la EFP dependen, en gran medida, de la nueva rectoría. A los tres candidatos les pregunté, en su visita de campaña al Programa de Psicología, qué pensaban de esta Escuela. Los tres coincidieron en alabar sus bondades y en declararse defensores de su fortalecimiento y continuidad. Pero una cosa es ser candidato urgido de votos y, otra, ser rector o rectora tratando de cumplir tantas promesas. Sin su apoyo decidido no habrá futuro para la Escuela. 
Respaldo que tiene que concretarse en un presupuesto económico creciente y en políticas que permitan las condiciones laborales básicas para que los profesores y las profesoras puedan participar.

El otro factor clave en el futuro de la EFP es el de la capacidad organizativa y argumentativa para no dejarla desaparecer, por parte del colectivo más comprometido con este proyecto. $\quad \mathrm{Si}$ estos educadores y educadoras no defienden el espacio ganado, con facilidad puede extinguirse. Junto a esta condición puedo sugerir las características fundamentales de su nuevo coordinador, que la dirigirá a partir de 2019. Más que la superficialidad de pertenecer a determinada Facultad, esta persona debería tener un conocimiento fuerte del campo pedagógico y del PEU, convicción por la importancia de los procesos para formar-nos en y desde la enseñanza superior, dedicación a la Escuela y responsabilidad con sus tareas. Y, sobre todo, que encarne con coherencia los discursos que la EFP promueve.

En estos primeros tres años la Escuela ha logrado posicionar, entre un significativo grupo de maestros y maestras, la reflexión en torno al PEU y a la formación pedagógica requerida para su aplicación en la enseñanza universitaria. Este avance en los procesos de formar-nos es imprescindible para pensar en la cualificación de la academia universitaria. Quienes trabajamos en la EFP reconocemos que hoy un profesor o una profesora universitaria, de tiempo completo, en una universidad pública con en PEU como el nuestro, no puede conformarse con poseer un cierto dominio sobre una parcela del conocimiento científico. Ni puede reducir su labor formativa a trasmitir unas cuantas técnicas y conceptos fragmentarios.

A propósito, Rodolfo LLinás sintetiza la esencia de su propuesta para que los profesores revolucionemos la enseñanza, con una frase que ataca la equivocada tradición escolar, memorística y abstracta. Se trata, ha dicho, de que los educadores nos concentremos en lograr que los estudiantes puedan: comprender en contexto. Y, claro está que, para poder conseguirlo, un profesor no podrá trabajar la comprensión desde metodologías pasivas y acríticas, carentes de creatividad e imaginación. Ni podrá poner en contexto lo que enseña, si sólo sabe algo de matemáticas, física, derecho o psicología, si no se forma en otros campos como los de la filosofía, las artes y la historia, que le permitan analizar y entender los grandes fenómenos y conflictos de la vida contemporánea. Esos acontecimientos atraviesan la educación y la vida misma de los jóvenes, los profesores y las profesoras que tenemos en las aulas.

Para formarnos en estos retos de la enseñanza superior, la USCO cuenta con un espacio, su EFP, que no debería desaparecer. Para trascender una concepción instrumental de la enseñanza, para recuperar la figura del profesor universitario como un humanista integral que trabaja para contribuir a la formación más amplia y plena de los universitarios, en sus dimensiones cognitivas, éticas, estéticas y políticas. Para hacer realidad el principio freireiano, según el cual, nadie se educa solo, ni la enseñanza es una acción de una única vía (del profesor al estudiante). Acción de una única vía (del profesor al estudiante). Es decir, que nos educamos en colectividad y que, al enseñar, también aprendemos, al colaborar para formar a otros, nos formamos. Por eso acudo a la expresión: formar-nos.

La EFP es, además, una oportunidad para combatir la banalización de la enseñanza universitaria, acuñada desde el modelo de universidad como empresa comercial. En nuestros días no sólo se banaliza el mal o el estudio, sino también la profesión de enseñar. Banalizar esta profesión es creer que ser profesor universitario es dictar tres cursos, desarrollar un proyecto de investigación y participar en las reuniones de un comité, con un enfoque insular disciplinar y con una actitud de indiferencia política sobre el devenir de la universidad y su contexto sociocultural.

Profesión y profesional vienen de profesar un saber en público. De profesarlo con convicción y alegría. El profesor integra y despliega mucho más que saber científico o técnico en su tarea de enseñar y formar. El conocimiento del maestro es vacío sin afecto y entusiasmo, estéril si el que lo pretende enseñar no lo practica, perverso si lo que se enseña se usa para ofender, ridiculizar o discriminar al estudiante. Peligroso si se ejerce con dogmatismo. El profesor es referente de 
humanidad (no modelo), ciudadanía crítica y desempeño profesional reflexivo. No sólo de investigar o dictar clases. El profesor es hoy (o debería ser), todavía, uno de los pocos responsables preparados para orientar juventudes. En este sentido, una profesora o un profesor universitario prudente saben que no pueden saberlo todo. Saben que es más lo que desconocen que lo que saben. Por eso también saben que tienen que esforzarse por saber cada día un poco más. Saben que saben con límites, con algunas zonas claras, otras claroscuras y demasiadas oscuras. Son profesores que, por experiencia y por formación, saben más que muchos de sus estudiantes, sin negarse la posibilidad de aprender de ellos. Si saben todo esto sabrán la necedad del dogmatismo.

Si saben que la enseñanza universitaria es una profesión exigente, no banal, entenderán que ella demanda una permanente indagación acerca de los conocimientos y prácticas que permiten su desarrollo, más allá del ¿qué enseño?, aparecerán en sus reflexiones interrogantes como: ¿a quién enseño?, ¿para qué lo hago?, ¿cómo y con qué?, ¿en qué contexto?, ¿qué tiene que ver lo que enseño con la vida personal y profesional del estudiante?, ¿qué relaciones tiene eso que enseño con otras disciplinas?, entre tantos otros. Si la enseñanza universitaria es una profesión digna, además, permitirá vivir de ella con decoro y nos exigirá, a cambio, una dedicación prioritaria a la institución que nos paga por ejercerla. Para todo esto la EFP es una oportunidad de elevado valor formativo.

Esta es mi mirada sobre la EFP, sus principales logros, dificultades y perspectivas. Es mi balance y, en cierta forma, mi rendición de cuentas, ahora que me retiro de la USCO. A la vicerrectora Isabel Cristina, a Alejandra Pérez González y al equipo de apoyo logístico, lo mismo que a los ciento cincuenta (150) profesores y profesoras, esporádicos y permanentes, participantes en la Escuela, les agradezco su confianza y las constructivas experiencias compartidas. 